\title{
Dissipative hydrodynamics for viscous relativistic fluids
}

\author{
Ulrich Heinz* and Huichao Song ${ }^{\dagger}$ \\ Department of Physics, Ohio State University, Columbus, Ohio 43210, USA \\ A. K. Chaudhuri ${ }^{\ddagger}$ \\ Variable Energy Cyclotron Centre, I/AF, Bidhan Nagar, Kolkata 700 064, India
}

(Received 4 October 2005; published 8 March 2006)

\begin{abstract}
Explicit equations are given for describing the space-time evolution of nonideal (viscous) relativistic fluids undergoing boost-invariant longitudinal and arbitrary transverse expansion. The equations are derived from the second-order Israel-Stewart approach which ensures causal evolution. Both azimuthally symmetric $(1+1)-$ dimensional and nonsymmetric $(2+1)$-dimensional transverse expansion are discussed. The latter provides the formal basis for the hydrodynamic computation of elliptic flow in relativistic heavy ion collisions including dissipative effects.
\end{abstract}

DOI: 10.1103/PhysRevC.73.034904

PACS number(s): 47.75.+f, 25.75.Ld

\section{INTRODUCTION}

Ideal fluid dynamics has been used successfully to predict the collective flow patterns in $\mathrm{Au}+\mathrm{Au}$ collisions at the BNL Relativistic Heavy-Ion Collider (RHIC) (for a review, see Ref. [1]). The ideal fluid description works well in almost central $\mathrm{Au}+\mathrm{Au}$ collisions near midrapidity at top RHIC energy, but gradually breaks down in more peripheral collisions, at forward rapidity, or at lower collision energies [2], indicating the onset of dissipative effects. To quantitatively describe such deviations from ideal fluid dynamics and to use the experimental data for the extraction of values or phenomenological limits for the transport coefficients of the hot and dense matter created during the collision requires the numerical implementation of dissipative relativistic fluid dynamics. A formulation of such a theory which avoids the long-standing problems of acausal signal propagation and other instabilities associated with the original relativistic fluid equations given by Eckart [3] and Landau and Lifshitz [4] has been known for almost 30 years [5]. Nevertheless, significant progress toward its numerical implementation has only been made very recently [6-9]. At this point, we are only at the very beginning of a program that will eventually apply viscous relativistic fluid dynamics to heavy-ion collision data. Existing numerical implementations are $(1+1)$-dimensional and can only describe cylindrically symmetric transverse expansion with boost-invariant longitudinal dynamics $[8,9]$. As we will show here, even the $(1+1)$ dimensional case still presents some open formal issues which we address in the present paper. The numerical codes are still in the process of being tested and will not be discussed here.

The paper is organized as follows: In Sec. II, we briefly review relativistic ideal fluid dynamics and the conditions for its applicability. While most of this is standard textbook material, it helps to establish notation and to better appreciate the differences in the nonideal case. In Sec. III, we discuss the nonideal fluid decomposition and introduce the dissipative

\footnotetext{
*E-mail: heinz@mps.ohio-state.edu

†E-mail: song@mps.ohio-state.edu

†E-mail: akc@veccal.ernet.in
}

flows (bulk and shear viscous pressure, heat conduction) and how they manifest themselves in the baryon current and energy-momentum tensor in the Eckart and Landau frames. Section IV deals with the derivation of equations to determine the evolution of these dissipative flows. We follow the treatment of Israel and Stewart [5] and discuss both the (acausal) first-order and (causal) second-order theories (this nomenclature will be explained in Sec. IV). While most of the material up to this point can already be found elsewhere $[5,6]$ (see also the beautiful lecture notes by Rischke in Ref. [10]), it is needed here for a self-contained presentation and for a critical discussion of some systematic expansion issues which we point out in Sec. IV B and which are of practical relevance. Section V contains the main results of this paper (with many technical details deferred to the Appendix), namely, complete sets of causal equations of motion for the dissipative transverse hydrodynamic expansion of systems undergoing boostinvariant longitudinal flow. The discussion of the azimuthally symmetric $(1+1)$-dimensional case in Sec. VA improves on the presentation given recently by Muronga and Rischke [8], while the equations for the nonsymmetric $(2+1)$-dimensional case in Sec. V B are original and, to our knowledge, have not been presented before. The concluding Sec. VI summarizes our results and gives some further discussion.

\section{IDEAL FLUID DYNAMICS}

Before explaining the structure of the equations for causal dissipative relativistic fluid dynamics, let us quickly review the case of ideal fluid dynamics. Any fluid dynamical approach starts from the conservation laws for the conserved charges and for energy-momentum,

$$
\begin{aligned}
\partial_{\mu} N_{i}^{\mu} & =0, \quad i=1, \ldots, k, \\
\partial_{\mu} T^{\mu \nu} & =0 .
\end{aligned}
$$

For simplicity, we will restrict ourselves to $k=1$ (say, $N_{\mu}=$ net baryon number current) and drop the index $i$ in Eq. (2.1). It must also ensure the second law of thermodynamics

$$
\partial_{\mu} S^{\mu} \geqslant 0
$$


where $S^{\mu}$ is the entropy current. Ideal fluid dynamics follows from these equations under the assumption of local thermal equilibrium; i.e., if the microscopic collision time scale is very much shorter than any macroscopic evolution time scale, then the underlying phase-space distribution $f(x, p)$ relaxes essentially instantaneously to a local equilibrium form (upper signs for fermions, lower signs for bosons)

$$
f_{\mathrm{eq}}(x, p)=\frac{1}{e^{[p \cdot u(x)+\mu(x)] / T(x)} \pm 1},
$$

where $u^{\mu}(x)$ is the local fluid velocity at point $x, \mu(x)$ is the local chemical potential associated with the conserved charge $N$ (it enters with opposite sign in the distribution $\bar{f}$ for antiparticles), and $T(x)$ is the local temperature. Plugging this into the kinetic theory definitions

$$
\begin{aligned}
N^{\mu}(x)= & \int \frac{d^{3} p}{E} p^{\mu}[f(x, p)-\bar{f}(x, p)], \\
T^{\mu \nu}(x)= & \int \frac{d^{3} p}{E} p^{\mu} p^{\nu}[f(x, p)+\bar{f}(x, p)], \\
S^{\mu}(x)= & -\int \frac{d^{3} p}{E} p^{\mu}[f(x, p) \ln f(x, p) \\
& \pm(1 \mp f(x, p)) \ln (1 \mp f(x, p))+(f \leftrightarrow \bar{f})],
\end{aligned}
$$

leads to the ideal fluid decompositions

$$
\begin{aligned}
N_{\mathrm{eq}}^{\mu} & =n u^{\mu}, \\
T_{\mathrm{eq}}^{\mu \nu} & =e u^{\mu} u^{\nu}-p \Delta^{\mu \nu}\left(\text { with } \Delta^{\mu \nu}=g^{\mu \nu}-u^{\mu} u^{\nu}\right), \\
S_{\mathrm{eq}}^{\mu} & =s u^{\mu},
\end{aligned}
$$

where the local net charge density $n$, energy density $e$, pressure $p$, and entropy density $s$ are given by the standard integrals over the thermal equilibrium distribution function in the local fluid rest frame and are related by the fundamental thermodynamic relation

$$
T s=p-\mu n+e .
$$

Inserting Eqs. (2.5)-(2.7) into Eqs. (2.1) and (2.2) yields the relativistic ideal fluid equations shown in Eqs. (2.12)-(2.14) below. Using Eq. (2.11) together with the Gibbs-Duhem relation $d p=s d T+n d \mu$, it is easy to prove that in the absence of shock discontinuities, these equations also conserve entropy, i.e., $\partial_{\mu} S^{\mu}=0$.

Note that the validity of the decompositions (2.5)-(2.7) only requires local momentum isotropy [i.e., in the local fluid rest frame the phase-space distribution reduces to a function of energy $E$ only, $f(x, p)=f(p \cdot u(x) ; T(x), \mu(x))]$, but does not require that the distribution function have the specific exponential form (2.4) which maximizes entropy. This may have relevance in situations where the time scale for local momentum isotropization is much shorter than for thermalization [11-13] (i.e., it is much easier to change the direction of the particles' momenta than their energies), with the macroscopic hydrodynamic time scale in between. In this case, the local microscopic states would not maximize entropy, and the relation (2.11) would not hold between the quantities $e, p, n$, and $s$ defined through Eqs. (2.5)-(2.10). Still, they would follow ideal fluid dynamical evolution since entropy production by microscopic kinetic energy-shifting processes would happen only on time scales that are large compared to the macroscopic evolution time scales. (Note that in the absence of such a clear separation of time scales, entropy production can not be neglected during the macroscopic evolution, and ideal fluid dynamics must be replaced by dissipative fluid dynamics.)

The ideal fluid equations read (with $\theta \equiv \partial \cdot u$ denoting the local expansion rate)

$$
\begin{aligned}
\dot{n} & =-n \theta, \\
\dot{e} & =-(e+p) \theta, \\
\dot{u}^{\mu} & =\frac{\nabla^{\mu} p}{e+p},
\end{aligned}
$$

where we decomposed the partial derivative $\partial^{\mu}=u^{\mu} D+\nabla^{\mu}$ into "longitudinal" and "transverse" components $D=u^{\nu} \partial_{v}$ and $\nabla^{\mu}=\Delta^{\mu \nu} \partial_{\nu}$, which in the local fluid rest frame reduce to the time derivative $\dot{f} \equiv D f$ and spatial gradient $\nabla f$. The first two equations describe the dilution of the local baryon and energy density due to the local expansion rate $\theta$, while the third describes the acceleration of the fluid by the spatial (in the local frame) pressure gradients, with the enthalpy $e+p$ acting as inertia. The five equations (2.12)-(2.14) for the six unknown functions $n, e, p, u^{\mu}$ (remember that $u^{\mu} u_{\mu}=1$ ) must be closed by supplying an equation of state (EOS) $p=$ $p(e, n)$.

\section{NONIDEAL FLUID DECOMPOSITION}

As the hydrodynamic evolution changes the local energy and baryon density, microscopic processes attempt to readjust the local phase-space distribution to corresponding new local temperatures and chemical potentials. If this does not happen fast enough, the phase-space distribution will start to deviate from its local equilibrium form (2.4): $f(x, p)=$ $f_{\text {eq }}(p \cdot u(x) ; T(x), \mu(x))+\delta f(x, p)$. The optimal values for the (readjusted) local temperature and chemical potential in the first term are fixed by imposing the "Landau matching conditions" [4]

$$
\begin{aligned}
u_{\mu} \delta T^{\mu \nu} u_{v} & =\int \frac{d^{3} p}{E}(u \cdot p)^{2} \delta f(x, p)=0, \\
u_{\mu} \delta N^{\mu} & =\int \frac{d^{3} p}{E}(u \cdot p) \delta f(x, p)=0 .
\end{aligned}
$$

The remaining deviations $\delta f$ from local equilibrium generate additional terms in the decompositions of $N^{\mu}, T^{\mu \nu}$, and $S^{\mu}$ :

$$
\begin{aligned}
& N^{\mu}=N_{\mathrm{eq}}^{\mu}+\delta N^{\mu}=n u^{\mu}+V^{\mu}, \\
& T^{\mu \nu}=T_{\mathrm{eq}}^{\mu \nu}+\delta T^{\mu \nu}=e u^{\mu} u^{v}-(p+\Pi) \Delta^{\mu \nu}+\pi^{\mu \nu} \\
& +W^{\mu} u^{v}+W^{v} u^{\mu} \text {, } \\
& S^{\mu}=S_{\mathrm{eq}}^{\mu}+\delta S^{\mu}=n u^{\mu}+\Phi^{\mu} .
\end{aligned}
$$

The new terms describe a baryon flow $V^{\mu}=\Delta^{\mu \nu} N_{v}$ in the local rest frame, an energy flow $W^{\mu}=\frac{e+p}{n} V^{\mu}+q^{\mu}$ (where $q^{\mu}$ is the "heat flow vector") in the local rest frame, the viscous bulk pressure $\Pi=-\frac{1}{3} \Delta_{\mu \nu} T^{\mu \nu}-p$ (which contributes to the trace of the energy momentum tensor), the traceless viscous shear pressure tensor $\pi^{\mu \nu}=$ $T^{\langle\mu \nu\rangle} \equiv\left[\frac{1}{2}\left(\Delta^{\mu \sigma} \Delta^{\nu \tau}+\Delta^{\nu \sigma} \Delta^{\mu \tau}\right)-\frac{1}{3} \Delta^{\mu \nu} \Delta^{\sigma \tau}\right] T_{\tau \sigma}$ (where the expression $\langle\mu \nu\rangle$ is shorthand for "traceless and transverse to 
$u_{\mu}$ and $u_{\nu}$," as defined by the projector in square brackets), and an entropy flow vector $\Phi^{\mu}$ in the local rest frame.

Inserting the decompositions (3.1), (3.4) into the conservation laws (2.1), (2.2) yields the nonideal fluid equations

$$
\begin{gathered}
\dot{n}=-n \theta-\nabla \cdot V+V \cdot \dot{u}, \\
\dot{e}=-(e+p+\Pi) \theta+\pi_{\mu \nu} \nabla^{\langle\mu} u^{\nu\rangle}-\nabla \cdot W+2 W \cdot \dot{u}, \\
(e+p+\Pi) \dot{u}^{\mu}=\nabla^{\mu}(p+\Pi)-\Delta^{\mu \nu} \nabla^{\sigma} \pi_{\nu \sigma}+\pi^{\mu \nu} \dot{u}_{v} \\
\quad-\left[\Delta^{\mu \nu} \dot{W}_{v}+W^{\mu} \theta+(W \cdot \nabla) u^{\mu}\right] .
\end{gathered}
$$

The matching conditions (3.1) and (3.2) leave the choice of the local rest frame velocity $u^{\mu}$ ambiguous. This ambiguity can be used to eliminate either $V^{\mu}$ from Eq. (3.3) ("Eckart frame," no baryon flow in the local rest frame [3]), in which case the energy flow reduces to the heat flow vector $W^{\mu}=q^{\mu}$, or $W^{\mu}$ from Eq. (3.4) ("Landau frame," no energy flow in the local rest frame [4]), in which case there is a nonzero baryon flow $V^{\mu}=-\frac{n}{e+p} q^{\mu}$ due to heat conduction in the local rest frame. (Intermediate frames are also possible, but yield no practical advantage.) For systems with vanishing net baryon number (as approximately realized in RHIC collisions) the Eckart frame is ill defined, and heat conduction disappears as an independent transport effect [14], so we will use the Landau frame. In this frame, for baryon-free systems with $n=0$ and no heat conduction, the nonideal fluid equations (3.6)-(3.8) simplify to

$$
\begin{aligned}
\dot{e} & =-(e+p+\Pi) \theta+\pi_{\mu \nu} \nabla^{\langle\mu} u^{\nu\rangle}, \\
(e+p+\Pi) \dot{u}^{\mu} & =\nabla^{\mu}(p+\Pi)-\Delta^{\mu \nu} \nabla^{\sigma} \pi_{\nu \sigma}+\pi^{\mu \nu} \dot{u}_{\nu} .
\end{aligned}
$$

The nonequilibrium decompositions (3.3)-(3.5) involve $1+3+5=9$ additional dynamical quantities, the "dissipative flows" $\Pi, q^{\mu}$, and $\pi^{\mu \nu}$ (the counting reflects their transversality to $u^{\mu}$ and the tracelessness of $\pi^{\mu \nu}$ ). This means that we need nine additional dynamical equations which should be compatible with the underlying transport theory for the nonequilibrium deviation $\delta f(x, p)$. For the baryon-free case without heat conduction, the number of additional equations needed reduces to six.

\section{KINETIC EQUATIONS FOR DISSIPATIVE FLOWS}

The key property of the kinetic equation governing the evolution of the phase-space distribution function $f=f_{\text {eq }}+$ $\delta f$ is that the collision term satisfies the second law of thermodynamics (2.3); i.e., entropy is produced until the system has reached a new state of local thermal equilibrium. We do not want to solve the kinetic theory; instead, we want to write a phenomenological macroscopic theory that is consistent with the constraints arising from the underlying kinetic theory, in particular the second law. The macroscopic theory will be constructed from an expansion of the entropy production rate in terms of the dissipative flows which themselves are proportional to the off-equilibrium deviation $\delta f$ of the phase-space distribution [5]. Assuming the latter to be small, $|\delta f| \ll\left|f_{\text {eq }}\right|$, this expansion will be truncated at some low order in the dissipative flows $\delta N^{\mu}, \delta T^{\mu \nu}$. The expansion will involve phenomenological expansion coefficients which, in principle, should be matched to the kinetic theory [5]. In practice, they will often be considered as phenomenological parameters to be adjusted to experimental data. The extracted values must then be checked for consistency with the entire approach by making sure that the dissipative corrections are indeed sufficiently small to justify truncation of the expansion a posteriori.

The equilibrium identity (2.11) can be rewritten as

$$
S_{\mathrm{eq}}^{\mu}=p(\alpha, \beta) \beta^{\mu}-\alpha N_{\mathrm{eq}}^{\mu}+\beta_{\nu} T_{\mathrm{eq}}^{\nu \mu}
$$

where $\alpha \equiv \frac{\mu}{T}, \beta \equiv \frac{1}{T}$, and $\beta_{\nu} \equiv \frac{u_{v}}{T}$. The most general offequilibrium generalization of this is [5]

$$
\begin{aligned}
S^{\mu} & \equiv S_{\mathrm{eq}}^{\mu}+\Phi^{\mu} \\
& =p(\alpha, \beta) \beta^{\mu}-\alpha N^{\mu}+\beta_{\nu} T^{\nu \mu}+Q^{\mu}\left(\delta N^{\mu}, \delta T^{\mu v}\right),
\end{aligned}
$$

where, in addition to the first-order contributions implicit in the second and third terms of the right-hand side, $Q^{\mu}$ includes terms which are second and higher order in the dissipative flows $\delta N^{\mu}$ and $\delta T^{\mu \nu}$. [Note that, by using the identity (2.11) between the equilibrium quantities, Eq. (4.2) can be written in the simpler-looking form $S^{\mu}=s u^{\mu}+\frac{q^{\mu}}{T}+Q^{\mu}$, but this is not helpful for calculating the entropy production rate.]

The form of the expansion (4.2) is constrained by the second law, $\partial_{\mu} S^{\mu} \geqslant 0$. To evaluate this constraint, it is useful to rewrite the Gibbs-Duhem relation $d p=s d T+n d \mu$ as

$$
\partial_{\mu}\left(p(\alpha, \beta) \beta^{\mu}\right)=N_{\text {eq }}^{\mu} \partial_{\mu} \alpha-T_{\text {eq }}^{\mu \nu} \partial_{\mu} \beta_{\nu} .
$$

With additional help from the conservation laws (2.1) and (2.2), the entropy production then becomes

$$
\partial_{\mu} S^{\mu}=-\delta N^{\mu} \partial_{\mu} \alpha+\delta T^{\mu \nu} \partial_{\mu} \beta_{\nu}+\partial_{\mu} Q^{\mu} .
$$

Using Eqs. (3.3) and (3.4) to express $\delta N^{\mu}$ and $\delta T^{\mu \nu}$ in terms of the scalar, vector, and tensor dissipative flows $\Pi, q^{\mu}$, and $\pi^{\mu \nu}$, and introducing the corresponding scalar, vector, and tensor thermodynamic forces (in terms of gradients of the thermodynamic equilibrium variables) which drive these dissipative flows, $X \equiv-\theta=-\nabla \cdot u, X^{v} \equiv \frac{\nabla^{v} T}{T}-\dot{u}^{v}=$ $-\frac{n T}{e+p} \nabla^{\nu}\left(\frac{\mu}{T}\right)$ [15], and $X^{\mu \nu} \equiv \nabla^{\langle\mu} u^{\nu\rangle}$ (note that $X^{\mu \nu}=X^{\langle\mu \nu\rangle}$ is traceless and transverse to $u$ ), the second law constraint can be further recast into

$$
T \partial_{\mu} S^{\mu}=\Pi X-q^{\mu} X_{\mu}+\pi^{\mu \nu} X_{\mu \nu}+T \partial_{\mu} Q^{\mu} \geqslant 0 .
$$

Note that the first three terms on the right-hand side are first order, whereas the last term is higher order in the dissipative flows.

\section{A. Standard dissipative fluid dynamics (first-order theory)}

The standard approach (which can be found, for example, in Ref. [4]) neglects the higher-order contributions and sets $Q^{\mu}=0$. The inequality (4.5) can then always be satisfied by postulating linear relationships between the dissipative flows and the thermodynamic forces,

$$
\begin{aligned}
\Pi & =-\zeta \theta, \\
q^{v} & =-\lambda \frac{n T^{2}}{e+p} \nabla^{v}\left(\frac{\mu}{T}\right), \\
\pi^{\mu \nu} & =2 \eta \nabla^{\langle\mu} u^{\nu\rangle},
\end{aligned}
$$


with positive transport coefficients $\zeta \geqslant 0$ (bulk viscosity), $\lambda \geqslant 0$ (heat conductivity), and $\eta \geqslant 0$ (shear viscosity):

$$
T \partial S=\frac{\Pi^{2}}{\zeta}-\frac{q^{\alpha} q_{\alpha}}{2 \lambda T}+\frac{\pi^{\alpha \beta} \pi_{\alpha \beta}}{2 \eta} \geqslant 0 .
$$

(The minus sign in front of the second term is necessary because $q^{\mu}$, being orthogonal to $u^{\mu}$, is spacelike, $q^{2}<0$.) These are the desired nine equations for the dissipative flows.

Unfortunately, using these relations in the hydrodynamic equations (3.6)-(3.8) leads to hydrodynamic evolution with acausal signal propagation: If in a given fluid cell at a certain time a thermodynamic force happens to vanish, the corresponding dissipative flow also stops instantaneously. This contradicts the fact that the flows result from the forces through microscopic scattering which involves relaxation on a finite albeit short kinetic time scale. To avoid this type of acausal behavior, one must keep $Q^{\mu}$.

\section{B. Second-order Israel-Stewart theory}

A causal theory of dissipative relativistic fluid dynamics is obtained by keeping $Q^{\mu}$ up to terms which are second order in the irreversible flows. For simplicity, we consider here only the baryon-free case $n=q^{\mu}=0$; for a general treatment, see Refs. [5,6]. One writes [5]

$$
Q^{\mu}=-\left(\beta_{0} \Pi^{2}+\beta_{2} \pi_{\nu \lambda} \pi^{\nu \lambda}\right) \frac{u^{\mu}}{2 T}
$$

(with phenomenological expansion coefficients $\beta_{0}, \beta_{2}$ ) and computes (after some algebra using similar techniques as before) the entropy production rate as

$$
\begin{aligned}
T \partial S & =\Pi\left[-\theta-\beta_{0} \dot{\Pi}-\Pi T \partial_{\mu}\left(\frac{\beta_{0} u^{\mu}}{2 T}\right)\right] \\
& +\pi^{\alpha \beta}\left[\nabla_{\langle\alpha} u_{\beta\rangle}-\beta_{2} \dot{\pi}_{\alpha \beta}-\pi_{\alpha \beta} T \partial_{\mu}\left(\frac{\beta_{2} u^{\mu}}{2 T}\right)\right] .
\end{aligned}
$$

From the expressions in the square brackets, we see that the thermodynamic forces $-\theta$ and $\nabla_{\langle\alpha} u_{\beta\rangle}$ are now selfconsistently modified by terms involving the time derivatives (in the local rest frame) of the irreversible flows $\Pi, \pi_{\alpha \beta}$. This leads to dynamical ("transport") equations for the latter. We can ensure the second law of thermodynamics by again writing the entropy production rate in the form (4.7) (without the middle term), which amounts to postulating

$$
\begin{aligned}
\dot{\Pi} & =-\frac{1}{\tau_{\Pi}}\left[\Pi+\zeta \theta+\Pi \zeta T \partial_{\mu}\left(\frac{\tau_{\Pi} u^{\mu}}{2 \zeta T}\right)\right] \\
& \approx-\frac{1}{\tau_{\Pi}}[\Pi+\zeta \theta], \\
\dot{\pi}_{\alpha \beta} & =-\frac{1}{\tau_{\pi}}\left[\pi_{\alpha \beta}-2 \eta \nabla_{\langle\alpha} u_{\beta\rangle}+\pi_{\alpha \beta} \eta T \partial_{\mu}\left(\frac{\tau_{\pi} u^{\mu}}{2 \eta T}\right)\right] \\
& \approx-\frac{1}{\tau_{\pi}}\left[\pi_{\alpha \beta}-2 \eta \nabla_{\langle\alpha} u_{\beta\rangle}\right] .
\end{aligned}
$$

Here we replaced the coefficients $\beta_{0,2}$ by the relaxation times $\tau_{\Pi} \equiv \zeta \beta_{0}$ and $\tau_{\pi} \equiv 2 \eta \beta_{2}$. In principle, both $\zeta, \eta$ and $\tau_{\Pi}, \tau_{\pi}$ should be calculated from the underlying kinetic theory. We will use them as phenomenological parameters, noting that for consistency the microscopic relaxation rates should be much larger than the local hydrodynamic expansion rate, $\tau_{\pi, \Pi} \theta \ll 1$.

Let us briefly discuss the approximation in the second equalities in Eqs. (4.10) and (4.11). We are using an expansion scheme for the entropy production rate in which the thermodynamic forces and irreversible flows are assumed to be small perturbations. The approximation in Eqs. (4.10), (4.11) neglects terms which are products of the irreversible flows with gradients of the thermodynamic equilibrium quantities which are of the same order as the thermodynamic forces. These terms are thus effectively of second order in small quantities and should, for consistency, be neglected relative to the other terms in the square brackets which are of first order. If one wants to keep them (as done by Muronga [6,8]), one should also keep third-order terms in the entropy flow vector $Q^{\mu}$ for consistency. Of course, where the thermodynamic forces and irreversible flows are really small, it should not matter whether we keep or drop these terms. In practice, however, one will use this approach when dissipative effects are expected to be significant, and the dropped terms may not be extremely small. In this case, we believe that dropping them is more consistent than keeping them.

There is another, more physical reason for dropping these terms: without them, Eqs. (4.10), (4.11) are relaxation equations which describe (in the local rest frame) exponential relaxation (on the time scales $\tau_{\pi, \Pi}$ ) of the irreversible flows to the values given by (4.6) in the first-order theory. However, if these terms are kept, one has instead equations of the form

$$
\begin{aligned}
\dot{\Pi} & =-\frac{1}{\tau_{\Pi}}\left[\Pi+\zeta \theta+\Pi \zeta \gamma_{\Pi}\right] \\
& =-\frac{1+\gamma_{\Pi} \zeta}{\tau_{\Pi}}\left[\Pi+\frac{\zeta}{1+\gamma_{\Pi} \zeta} \theta\right]=-\frac{1}{\tau_{\Pi}^{\prime}}\left[\Pi+\zeta^{\prime} \theta\right],
\end{aligned}
$$

[where $\gamma_{\Pi} \equiv T \partial_{\mu}\left(\frac{\tau_{\Pi} u^{\mu}}{2 \zeta T}\right)$ ], and similarly for the shear pressure tensor. One sees that the (small) last term in Eq. (4.10) modifies both the kinetic relaxation time and the viscosity by an amount $\sim \gamma_{\Pi}$ which involves the macroscopic expansion rate $\partial_{\mu} u^{\mu}$. This contradicts the intuitive expectation that the effective relaxation time and viscosity which control the kinetic evolution of $\Pi$ should be expressible through integrals of the kinetic collision term that involve only microscopic physics (cross sections, local densities, etc.).

In the second-order Israel-Stewart formalism, one thus solves the dissipative hydrodynamic equations (3.6)-(3.8) simultaneously with the kinetic relaxation equations (4.10), (4.11) for the irreversible flows. Let us now look at these equations in more detail when expressed in a global coordinate system (and not in local rest frame coordinates as done up to now).

\section{TRANSVERSE EXPANSION DYNAMICS IN SYSTEMS WITH LONGITUDINAL BOOST INVARIANCE}

We are restricting our discussion to systems with longitudinal boost invariance. With this approximation, we can describe the transverse expansion in very high energy heavy-ion colli- 
sions in a domain near midrapidity. Boost-invariant systems are conveniently described in $(\tau, x, y, \eta)$ coordinates, where $\tau=$ $\sqrt{t^{2}-z^{2}}$ is longitudinal proper time, $\eta=\frac{1}{2} \ln [(t+z) /(t-z)]$ is space-time rapidity, and $\boldsymbol{r}_{\perp}=(x, y)$ are the usual Cartesian coordinates in the plane transverse to the beam direction $z$. Boost-invariant systems are then characterized by macroscopic observables which are independent of $\eta$ and by phase-space distributions which depend only on the difference $Y-\eta$ (where $Y=\frac{1}{2} \ln \left[\left(E+p_{z}\right) /\left(E-p_{z}\right)\right]$ is the momentum-space rapidity of a particle with longitudinal momentum $p_{z}$ and energy $E)$. We denote two-dimensional vectors in the transverse plane by $\boldsymbol{r}_{\perp}, \boldsymbol{v}_{\perp}, \nabla_{\perp}$, etc., and generally use lower-case Latin letters to denote vector and tensor components in this curvilinear space-time coordinate system. The metric tensor in this coordinate system reads

$$
\begin{aligned}
& g^{m n}=\operatorname{diag}\left(1,-1,-1,-1 / \tau^{2}\right), \\
& g_{m n}=\operatorname{diag}\left(1,-1,-1,-\tau^{2}\right) .
\end{aligned}
$$

The flow velocity is parametrized as

$$
u^{m}=\gamma_{\perp}\left(1, v_{x}, v_{y}, 0\right)=\gamma_{\perp}\left(1, \boldsymbol{v}_{\perp}, 0\right),
$$

where

$$
\gamma_{\perp}=\frac{1}{\sqrt{1-v_{\perp}^{2}}}=\frac{1}{\sqrt{1-v_{x}^{2}-v_{y}^{2}}},
$$

with vanishing flow component $u^{\eta}$ in $\eta$ direction and transverse flow velocity $\boldsymbol{v}_{\perp}\left(\tau, \boldsymbol{r}_{\perp}\right)$. For vectors and tensors, the usual Cartesian derivatives $\partial_{\mu}$ must be replaced by covariant derivatives, denoted by semicolons,

$$
\begin{aligned}
\partial_{\mu} j^{\nu} & \rightarrow j_{; m}^{n}=\partial_{m} j^{n}+\Gamma_{m k}^{n} j^{k}, \\
\partial_{\mu} T^{\nu \lambda} & \rightarrow T_{; m}^{n l}=\partial_{m} T^{n l}+\Gamma_{m k}^{n} T^{k l}+T^{n k} \Gamma_{k m}^{l},
\end{aligned}
$$

where $\Gamma_{j k}^{i}=\frac{1}{2} g^{i m}\left(\partial_{j} g_{k m}+\partial_{k} g_{m j}-\partial_{m} g_{j k}\right)$ are the Christoffel symbols. The only nonvanishing components of $\Gamma_{j k}^{i}$ are

$$
\Gamma_{\eta \tau}^{\eta}=\Gamma_{\tau \eta}^{\eta}=\frac{1}{\tau}, \quad \Gamma_{\eta \eta}^{\tau}=\tau .
$$

The time derivative in the local comoving frame and the local expansion rate are thus computed as

$$
\begin{aligned}
D & =u \cdot \partial=\gamma_{\perp}\left(\partial_{\tau}+\boldsymbol{v}_{\perp} \cdot \nabla_{\perp}\right), \\
\theta & =\partial \cdot u=\frac{1}{\tau} \partial_{\tau}\left(\tau \gamma_{\perp}\right)+\nabla_{\perp} \cdot\left(\gamma_{\perp} \boldsymbol{v}_{\perp}\right) .
\end{aligned}
$$

If the expanding system has additionally azimuthal symmetry around the beam direction (for example, central collisions between spherically symmetric nuclei), it is advantageous to replace the Cartesian transverse coordinates $(x, y)$ by polar coordinates $(r, \phi)$ since macroscopic quantities are then $\phi$ independent, that is,

$$
\begin{aligned}
x^{m} & =(\tau, r, \phi, \eta), \\
g^{m n} & =\operatorname{diag}\left(1,-1,-1 / r^{2},-1 / \tau^{2}\right), \\
g_{m n} & =\operatorname{diag}\left(1,-1,-r^{2},-\tau^{2}\right) .
\end{aligned}
$$

This leads to the following additional [to Eq. (5.5)] nonvanishing Christoffel symbols

$$
\Gamma_{\phi r}^{\phi}=\Gamma_{r \phi}^{\phi}=+\frac{1}{r}, \quad \Gamma_{\phi \phi}^{r}=-r .
$$

The flow velocity now simplifies to

$$
u^{m}=\gamma_{r}\left(1, v_{r}, 0,0\right), \quad \text { with } \quad \gamma_{r}=\frac{1}{\sqrt{1-v_{r}^{2}}},
$$

radial transverse flow velocity $\boldsymbol{v}_{\perp}=v_{r}(\tau, r) \boldsymbol{e}_{r}$, and vanishing flow components $u^{\phi}$ and $u^{\eta}$. Correspondingly, the time derivative in the local comoving frame and the local expansion rate reduce to

$$
\begin{aligned}
D & =u \cdot \partial=\gamma_{r}\left(\partial_{\tau}+v_{r} \partial_{r}\right), \\
\theta & =\partial \cdot u=\frac{1}{\tau} \partial_{\tau}\left(\tau \gamma_{r}\right)+\frac{1}{r} \partial_{r}\left(r v_{r} \gamma_{r}\right) .
\end{aligned}
$$

We will now treat the azimuthally symmetric and nonsymmetric cases separately.

\section{A. (1+1)-dimensional viscous hydrodynamics: Azimuthally symmetric case}

Due to azimuthal symmetry and longitudinal boost invariance, the $n=\phi$ and $n=\eta$ components of the equations of motion $T_{; m}^{m n}=0$ are redundant. Using the results of the appendix Sec. 1 , the $n=\tau$ and $n=r$ components can be written as

$$
\begin{gathered}
\frac{1}{\tau} \partial_{\tau}\left(\tau T^{\tau \tau}\right)+\frac{1}{r} \partial_{r}\left(r T^{\tau r}\right)=-\frac{p+\Pi+\tau^{2} \pi^{\eta \eta}}{\tau}, \\
\frac{1}{\tau} \partial_{\tau}\left(\tau T^{\tau r}\right)+\frac{1}{r} \partial_{r}\left(r\left(T^{\tau r} v_{r}+\mathcal{P}_{r}\right)\right)=+\frac{p+\Pi+r^{2} \pi^{\phi \phi}}{r} .
\end{gathered}
$$

With the shorthand notations $\tilde{T}^{m n}=r \tau T^{m n}, \tilde{\mathcal{P}}_{r}=r \tau \mathcal{P}_{r}$, and $\tilde{v}_{r}=\frac{\tilde{T}^{\tau r}}{\tilde{T}^{\tau \tau}}=\frac{T^{\tau r}}{T^{\tau \tau}}$ these are brought into standard (Cartesian) form

$$
\begin{aligned}
\partial_{\tau} \tilde{T}^{\tau \tau}+\partial_{r}\left(\tilde{v}_{r} \tilde{T}^{\tau \tau}\right) & =-r\left(p+\Pi+\tau^{2} \pi^{\eta \eta}\right), \\
\partial_{\tau} \tilde{T}^{\tau r}+\partial_{r}\left(v_{r} \tilde{T}^{\tau r}+\tilde{\mathcal{P}}_{r}\right) & =\tau\left(p+\Pi+r^{2} \pi^{\phi \phi}\right) .
\end{aligned}
$$

The corresponding transport equations for the dissipative fluxes read [using the explicit expressions (A11) for the shear tensor from appendix]

$$
\begin{aligned}
\left(\partial_{\tau}+v_{r} \partial_{r}\right) \pi^{\eta \eta} & =-\frac{1}{\gamma_{r} \tau_{\pi}}\left[\pi^{\eta \eta}-\frac{2 \eta}{\tau^{2}}\left(\frac{\theta}{3}-\frac{\gamma_{r}}{\tau}\right)\right] \\
\left(\partial_{\tau}+v_{r} \partial_{r}\right) \pi^{\phi \phi} & =-\frac{1}{\gamma_{r} \tau_{\pi}}\left[\pi^{\phi \phi}-\frac{2 \eta}{r^{2}}\left(\frac{\theta}{3}-\frac{\gamma_{r} v_{r}}{r}\right)\right] \\
\left(\partial_{\tau}+v_{r} \partial_{r}\right) \Pi & =-\frac{1}{\gamma_{r} \tau_{\Pi}}[\Pi+\zeta \theta] .
\end{aligned}
$$

Similar equations were derived in Ref. [8] [with extra terms, however, resulting from the higher-order corrections in Eqs. (4.10) and (4.11) which we argued should be neglected]. These equations can be solved with the code LCPFCT [16], using subroutine LCPFCT for Eq. (5.15) and subroutine CNVFCT 
for Eq. (5.16). First attempts at a numerical solution have been reported in Refs. [8,9], but a number of open questions remain at this point, and we leave a detailed discussion of the numerical aspects to a subsequent publication.

The hydrodynamic equations require the equation of state $p(e)$ for closure; i.e., after each transport step in time, we must extract at each spatial grid point the boost velocity $v_{r}$ between the global and local rest frames and the local energy density $e$ from the dynamical variables $T^{\tau \tau}$ and $T^{\tau r}$. Equations (A8) give the energy density as

$$
e=T^{\tau \tau}-v_{r} T^{\tau r},
$$

where the radial velocity $v_{r}$ must be extracted from the implicit equation

$$
v_{r}=\frac{T^{\tau r}}{T^{\tau \tau}+p\left(e=T^{\tau \tau}-v_{r} T^{\tau r}\right)+\Pi-r^{2} \pi^{\phi \phi}-\tau^{2} \pi^{\eta \eta}}
$$

by a one-dimensional zero search. This is still the same degree of numerical complexity as in the ideal fluid case [10]; we will see in the next subsection, however, that this part of the problem becomes numerically more involved for dissipative hydrodynamics without azimuthal symmetry.

\section{B. (2+1)-dimensional viscous hydrodynamics with longitudinal boost invariance}

In the absence of azimuthal symmetry, only the $n=\eta$ component of the equations of motion $T^{m n}{ }_{; m}=0$ is redundant (due to boost invariance). Using the relations (A21) from the appendix, the $n=\tau, x, y$ components can be written as

$$
\begin{aligned}
& \frac{1}{\tau} \partial_{\tau}\left(\tau T^{\tau \tau}\right)+\partial_{x} T^{\tau x}+\partial_{y} T^{\tau y}=-\frac{p+\Pi+\tau^{2} \pi^{\eta \eta}}{\tau}, \\
& \frac{1}{\tau} \partial_{\tau}\left(\tau T^{\tau x}\right)+\partial_{x}\left(\left(T^{\tau x}-\pi^{\tau x}\right) v_{x}\right)+\partial_{y}\left(\left(T^{\tau x}-\pi^{\tau x}\right) v_{y}\right) \\
& \quad=-\partial_{x}\left(p+\Pi+\pi^{x x}\right)-\partial_{y} \pi^{x y}, \\
& \frac{1}{\tau} \partial_{\tau}\left(\tau T^{\tau y}\right)+\partial_{x}\left(\left(T^{\tau y}-\pi^{\tau y}\right) v_{x}\right)+\partial_{y}\left(\left(T^{\tau y}-\pi^{\tau y}\right) v_{y}\right) \\
& \quad=-\partial_{x} \pi^{x y}-\partial_{y}\left(p+\Pi+\pi^{y y}\right) .
\end{aligned}
$$

The further manipulation of these equations depends on our choice of independent shear pressure components as dynamical variables. In the following two subsections, we explore two different choices, each with its own advantages and disadvantages.

\section{Choosing $\pi^{\tau \tau}, \Delta=\pi^{x x}-\pi^{y y}$, and $\pi^{\eta \eta}$ as independent dynamical variables}

In the first approach, we select $\pi^{\tau \tau}, \pi^{\eta \eta}$, and the difference $\Delta=\pi^{x x}-\pi^{y y}$ as independent dynamical components of the shear pressure tensor. The last choice has the advantage that it vanishes in the azimuthally symmetric case, thereby automatically reducing the number of independent dynamical variables. The choice of $\pi^{\tau \tau}$ instead of the orthogonal combination $\Sigma=\pi^{x x}+\pi^{y y}$ is a matter of taste and not essential. They are related by Eq. (A19), $\pi^{\tau \tau}=\Sigma+\tau^{2} \pi^{\eta \eta}$.
Introducing the shorthand notations $\tilde{T}^{m n}=\tau T^{m n}$ (without the factor $r$ this time), $\tilde{\pi}^{m n}=\tau \pi^{m n}, \tilde{p}=\tau p, \tilde{\Pi}=$ $\tau \Pi$, and $\tilde{v}_{i}=\frac{\tilde{T}^{\tau i}}{\tilde{T}^{\tau \tau}}=\frac{T^{\tau i}}{T^{\tau \tau}}$ for $i=x, y$, and following the procedure in the appendix, Eqs. (5.19) can be recast into standard form

$$
\begin{gathered}
\partial_{\tau} \tilde{T}^{\tau \tau}+\partial_{x}\left(\tilde{T}^{\tau x} \tilde{v}_{x}\right)+\partial_{y}\left(\tilde{T}^{\tau y} \tilde{v}_{y}\right)=-\left(p+\Pi+\tau^{2} \pi^{\eta \eta}\right), \\
\partial_{\tau} \tilde{T}^{\tau x}+\partial_{x}\left(\left(\tilde{T}^{\tau x}-\tilde{\pi}^{\tau x}\right) v_{x}\right)+\partial_{y}\left(\left(\tilde{T}^{\tau x}-\tilde{\pi}^{\tau x}\right) v_{y}\right) \\
=-\partial_{x}\left(\tilde{p}+\tilde{\Pi}+\tilde{\pi}^{x x}\right)-\partial_{y} \tilde{\pi}^{x y}, \\
\partial_{\tau} \tilde{T}^{\tau y}+\partial_{x}\left(\left(\tilde{T}^{\tau y}-\tilde{\pi}^{\tau y}\right) v_{x}\right)+\partial_{y}\left(\left(\tilde{T}^{\tau y}-\tilde{\pi}^{\tau y}\right) v_{y}\right) \\
=-\partial_{x} \tilde{\pi}^{x y}-\partial_{y}\left(\tilde{p}+\tilde{\Pi}+\tilde{\pi}^{y y}\right),
\end{gathered}
$$

where we have resisted inserting the lengthy explicit expressions (A22) for $\pi^{\tau x}, \pi^{\tau y}, \pi^{x y}, \pi^{x x}$, and $\pi^{y y}$. Note that the latter involve the velocities $v_{x}$ and $v_{y}$; so, to evaluate the sources at time step $n$ which drive the propagation to time step $n+1$, we must explicitly solve for the velocities $v_{x, y}\left(\boldsymbol{r}_{\perp}\right)$ at time step $n$. We will return to this issue momentarily.

The transport equations to be solved together with Eqs. (5.20) are (see appendix)

$$
\begin{aligned}
\left(\partial_{\tau}+v_{x} \partial_{x}+v_{y} \partial_{y}\right) \pi^{\eta \eta} & =-\frac{1}{\gamma_{\perp} \tau_{\pi}}\left(\pi^{\eta \eta}-2 \eta \sigma^{\eta \eta}\right), \\
\left(\partial_{\tau}+v_{x} \partial_{x}+v_{y} \partial_{y}\right) \pi^{\tau \tau} & =-\frac{1}{\gamma_{\perp} \tau_{\pi}}\left(\pi^{\tau \tau}-2 \eta \sigma^{\tau \tau}\right), \\
\left(\partial_{\tau}+v_{x} \partial_{x}+v_{y} \partial_{y}\right) \Delta & =-\frac{1}{\gamma_{\perp} \tau_{\pi}}\left(\Delta-2 \eta \sigma^{\Delta}\right), \\
\left(\partial_{\tau}+v_{r} \partial_{r}\right) \Pi & =-\frac{1}{\gamma_{\perp} \tau_{\Pi}}(\Pi+\zeta \theta),
\end{aligned}
$$

where $\sigma^{\eta \eta}, \sigma^{\tau \tau}$, and $\sigma^{\Delta}$ are given by Eqs. (A23).

In order to compute the pressure $p$ from the equation of state $p(e)$, we calculate from the dynamical variables $T^{\tau \tau}, T^{\tau x}$, and $T^{\tau y}$ the energy density by combining Eqs. (A21a)-(A21c):

$$
e=T^{\tau \tau}-v_{x} T^{\tau x}-v_{y} T^{\tau y} .
$$

(Note that all viscous pressures cancel in this relation.) This requires the velocities $v_{x}$ and $v_{y}$ which are given (implicitly!) by

$$
\begin{aligned}
& v_{x}=\frac{T^{\tau x}-\pi^{\tau x}}{T^{\tau \tau}+p(e)+\Pi-\pi^{\tau \tau}}, \\
& v_{y}=\frac{T^{\tau y}-\pi^{\tau y}}{T^{\tau \tau}+p(e)+\Pi-\pi^{\tau \tau}} .
\end{aligned}
$$

Since the vectors $\left(T^{\tau x}, T^{\tau y}\right)$ and $\left(\pi^{\tau x}, \pi^{\tau y}\right)$ are not parallel to each other, the direction of the flow velocity $\boldsymbol{v}_{\perp}=\left(v_{x}, v_{y}\right)$ is no longer given by the direction of $\left(T^{\tau x}, T^{\tau y}\right)$ as is the case in ideal fluid dynamics [10], and Eqs. (5.23) can no longer be reduced to a simple one-dimensional zero search. Instead, one must simultaneously iterate two equations, one for the 
magnitude of the transverse velocity,

$$
v_{\perp}^{2}=\frac{T^{\tau \tau}-\pi^{\tau \tau}-e}{T^{\tau \tau}-\pi^{\tau \tau}+p(e)+\Pi},
$$

which is easily verified from Eq. (A21a), and one for the azimuthal angle of the velocity vector $\phi_{v}=\tan ^{-1}\left(\frac{v_{y}}{v_{x}}\right)$. The latter is obtained by writing $v_{x}=v_{\perp} \cos \phi_{v} \equiv v_{\perp} z$, and $v_{y}=$ $v_{\perp} \sin \phi_{v}=v_{\perp} \sqrt{1-z^{2}}$, dividing the two equations (5.23) by each other, and inserting Eqs. (A22a), (A22b) to obtain

$$
\begin{aligned}
& z^{2}\left[2 v_{\perp} \sqrt{1-z^{2}} T^{\tau y}-\pi^{\tau \tau}\right. \\
& \left.+\frac{v_{\perp}^{2}}{2}\left(\left(\pi^{\tau \tau}-\tau^{2} \pi^{\eta \eta}\right)\left(2 z^{2}-1\right)+\frac{\Delta}{2}\right)\right] \\
& -\left(1-z^{2}\right)\left[2 v_{\perp} z T^{\tau x}-\pi^{\tau \tau}\right. \\
& \left.\quad-\frac{v_{\perp}^{2}}{2}\left(\left(\pi^{\tau \tau}-\tau^{2} \pi^{\eta \eta}\right)\left(2 z^{2}-1\right)+\frac{\Delta}{2}\right)\right]=0 .
\end{aligned}
$$

Since Eq. (5.24) requires knowledge of the direction of $\boldsymbol{v}_{\perp}$ on the right-hand side because of Eq. (5.22), and Eq. (5.25) requires knowledge of $v_{\perp}$, these two equations cannot be decoupled, and the iteration problem is genuinely two-dimensional. This is of serious concern since this problem must be solved at every spatial grid point after each time step which makes it numerically very expensive.

\section{Choosing $\pi^{\tau \tau}, \pi^{\tau x}, \pi^{\tau y}$, and $\pi^{\eta \eta}$ as independent dynamical variables}

This problem can be avoided if, instead of $\Delta, \pi^{\tau x}$ and $\pi^{\tau y}$ are kept as dynamical variables which are directly evolved in time via their own kinetic transport equations. Defining the two-dimensional vector $\boldsymbol{M}=\left(M_{x}, M_{y}\right) \equiv$ $\left(T^{\tau x}-\pi^{\tau x}, T^{\tau y}-\pi^{\tau y}\right.$ ) (which is just the transverse momentum density vector without the shear pressure contributions), we see from Eqs. (5.23) that $\boldsymbol{v}_{\perp}$ and $\boldsymbol{M}$ are parallel, $\boldsymbol{v}_{\perp} \cdot$ $\boldsymbol{M}=v_{\perp} M$ with $M=\sqrt{M_{x}^{2}+M_{y}^{2}}$. Furthermore, introducing $M_{0} \equiv T^{\tau \tau}-\pi^{\tau \tau}$, Eq. (5.22) can with the help of Eq. (A20c) be rewritten as

$$
e=M_{0}-\boldsymbol{v}_{\perp} \cdot \boldsymbol{M}=M_{0}-v_{\perp} M,
$$

which requires knowledge of only the magnitude of $v_{\perp}$. The latter can then be obtained by a normal one-dimensional zero search from Eq. (5.24), which can also be written as

$$
v_{\perp}=\frac{M}{M_{0}+p\left(e=M_{0}-v_{\perp} M\right)+\Pi},
$$

and the velocity components are reconstructed from

$$
v_{x}=v_{\perp} \frac{M_{x}}{M}, \quad v_{x}=v_{\perp} \frac{M_{y}}{M} .
$$

Note that this procedure requires direct knowledge of $\pi^{\tau \tau}, \pi^{\tau x}$, and $\pi^{\tau y}$ at all spatial grid points at each time step, so $\pi^{\tau x}$ and $\pi^{\tau y}$ cannot be computed from the constraints (A22a) and (A22b) (for which velocities $v_{x}$ and $v_{y}$ would need to be already known). On the other hand, $\pi^{\tau \tau}, \pi^{\tau x}$, and $\pi^{\tau y}$ are not independent, but related by Eq. (A20c). The suggested procedure requires independently propagating all three of these shear pressure components via kinetic transport equations [which, of course, should accurately preserve the constraint (A20c) if correctly implemented numerically]. Therefore, we have to solve one more kinetic transport equation (involving one physically redundant component) than in the procedure of Sec. VB 1. Instead of the three independent kinetic transport equations (5.21a)-(5.21c), we have to solve four equations for $\pi^{\tau \tau}, \pi^{\tau x}, \pi^{\tau y}$, and $\pi^{\eta \eta}$.

The set of equations to be solved simultaneously in this approach is given by Eqs. (5.20) and (5.21a,b,d), plus the following two equations:

$$
\begin{aligned}
& \left(\partial_{\tau}+v_{x} \partial_{x}+v_{y} \partial_{y}\right) \pi^{\tau x}=-\frac{1}{\gamma_{\perp} \tau_{\pi}}\left(\pi^{\tau x}-2 \eta \sigma^{\tau x}\right), \\
& \left(\partial_{\tau}+v_{x} \partial_{x}+v_{y} \partial_{y}\right) \pi^{\tau y}=-\frac{1}{\gamma_{\perp} \tau_{\pi}}\left(\pi^{\tau y}-2 \eta \sigma^{\tau y}\right) .
\end{aligned}
$$

The shear tensor components required here are given in Eqs. (A25).

Is this approach more economical than the two-dimensional zero search from the previous subsection? We believe so. If a one-dimensional zero search requires $N$ iterations, each with $K$ algebraic manipulations, a two-dimensional zero search would require $\mathcal{O}\left(N^{2} K K^{\prime}\right)$ algebraic manipulations at each spatial grid point and time step. Solving instead an additional kinetic transport equation for, say, $\pi^{\tau x}$ requires $\mathcal{O}\left(K_{s} K_{t}\right)$ algebraic manipulations at each time and grid point, where $K_{s}$ is the number of algebraic steps required to evaluate the source $\sigma^{\tau x}$ and $K_{t}$ is the number of algebraic steps involved in the time evolution algorithm. Taking the number $K^{\prime}$ of manipulations required to evaluate Eq. (5.25) to be comparable to $K_{s}$ should make solving the extra kinetic transport equation numerically less expensive since we expect $K_{t}$ to be significantly smaller than $N^{2} K$. The actual numerical implementation will tell whether this expectation is borne out. In any case, dissipative hydrodynamics is considerably more expensive than ideal fluid dynamics, and efficient coding will be required.

\section{SUMMARY}

In this paper, we derived explicit equations of motion in a form that makes them directly amenable to publicly available transport algorithms [16] for a causal theory of dissipative hydrodynamic evolution for relativistic viscous fluids such as those created in relativistic heavy-ion collisions. In doing so, we followed the pioneering work of Israel and Stewart [5], which was recently brought to wider attention and worked out in greater detail by Muronga [6]. Our treatment is still not completely general in that it continues to assume boost-invariant expansion along the beam direction (thereby reducing the spatial dimensionality of the problem by 1), but it goes beyond the existing literature [6,7] by allowing for arbitrary transverse expansion, without the additional restriction of azimuthal symmetry around the beam direction. It thus provides the formal basis for a numerical calculation of elliptic flow in relativistic heavy-ion collisions including dissipative effects. Such calculations will be needed for the 
phenomenological determination of the viscosity of the quarkgluon plasma from heavy-ion collision data.

We have also added to the discussion in Ref. [8] of azimuthally symmetric $(1+1)$-dimensional viscous hydrodynamics by reanalyzing the Israel-Stewart approach [5] as presented in Ref. [6] and pointing out a systematic issue with the expansion of the entropy current in terms of higher-order terms in the dissipative fluxes. The practical importance of the improved truncation scheme suggested here will only be fully assessed once the numerical implementation of our equations [9] has been thoroughly tested and becomes available for systematic investigations.

The simplification of the problem resulting from azimuthal symmetry, by exploiting polar coordinates in the transverse plane, is significant. The effects of viscosity can be subsumed into an effective radial pressure, leaving intact the diagonal structure of the energy-momentum tensor in the local rest frame. As a consequence, the numerically critical problem of extracting at each time step from the dynamical components of $T^{\mu \nu}$ the local flow velocity and energy density in order to compute the pressure from the equation of state remains one-dimensional, i.e., of the same complexity as for ideal fluids. The additional complexity resulting from dissipation thus resides entirely in the need for solving, together with the two hydrodynamic evolution equations, three additional kinetic transport equations for the bulk viscous pressure and for two components of the shear viscous pressure.

For the general situation without azimuthal symmetry, the use of polar coordinates (with their coordinate singularity at $r_{\perp}=0$ ) only complicates matters. It provides no help toward solving the now in general two-dimensional self-consistency problem associated with the extraction of the flow velocity and local energy density from the dynamical variables. We, therefore, use Cartesian coordinates in the transverse plane, as has been the tradition in $(2+1)$-dimensional ideal fluid dynamics. Unfortunately, this choice eliminates the possibility of coding the equations in such a way that the code automatically takes full advantage of all the simplifications resulting from azimuthal symmetry when handed an azimuthally symmetric problem. Azimuthally symmetric $(1+1)$-dimensional expansion and asymmetric $(2+1)$-dimensional expansion require differently optimized algorithms.

Using Cartesian transverse coordinates, we found a nice way of avoiding the above-mentioned two-dimensional nature of the numerically critical iteration problem for the local energy density, by increasing the set of kinetic transport equations not by 1 , but by 2 relative to the azimuthally symmetric case. By keeping one of the redundant components of the shear pressure tensor as a dynamical variable, we can again bring the iteration problem for the local energy density into scalar form. We believe that the expense for solving an additional transport equation, although not negligible, is less than that required for coping with a two-dimensional iteration problem at each time step at all spatial grid points.

Compared to $(2+1)$-dimensional ideal fluid dynamics, dissipative dynamics generates more complicated source terms for the three independent hydrodynamic evolution equations and requires the additional simultaneous solution of five kinetic transport equations, one for the bulk viscous pressure and four for shear viscous pressure components (one of them being physically, but not algorithmically redundant). Altogether, the resulting increase in numerical complexity (compared to the ideal fluid case) is probably less than an order of magnitude. Given the increase in computer speed and power experienced during the past decade, this should be manageable.

\section{ACKNOWLEDGMENTS}

We thank A. Muronga for directing us to the LCPFCT code. The work of U.H. was supported by the U.S. Department of Energy under Contract DE-FG02-01ER41190.

\section{APPENDIX: SHEAR TENSOR AND VISCOUS PRESSURE TENSOR COMPONENTS}

\section{Azimuthally symmetric systems}

For azimuthally symmetric systems, we use polar coordinates in the transverse plane: $x^{m}=(\tau, r, \phi, \eta)$. In this coordinate system, the global frame and local fluid rest frame are connected by a radial boost with velocity $\boldsymbol{v}=v_{r}(r, \tau) \boldsymbol{e}_{r}$. Introducing the fluid rapidity $y_{r}=\tanh ^{-1} v_{r}$ such that $\gamma_{r}=$ $\cosh y_{r}$ and $\gamma_{r} v_{r}=\sinh v_{r}$, the corresponding Lorentz transformation matrix is given by

$$
\Lambda_{n}^{m}\left(v_{r}\right)=\left(\begin{array}{cccc}
\cosh y_{r} & \sinh y_{r} & 0 & 0 \\
\sinh y_{r} & \cosh y_{r} & 0 & 0 \\
0 & 0 & 1 & 0 \\
0 & 0 & 0 & 1
\end{array}\right) .
$$

The projector transverse to the flow vector $u^{m}$ takes the form

$$
\Delta_{n}^{m}=\left(\begin{array}{cccc}
-\gamma_{r}^{2} v_{r}^{2} & \gamma_{r}^{2} v_{r} & 0 & 0 \\
-\gamma_{r}^{2} v_{r} & \gamma_{r}^{2} & 0 & 0 \\
0 & 0 & 1 & 0 \\
0 & 0 & 0 & 1
\end{array}\right) .
$$

(Note that this differs from $\Delta_{m}{ }^{n}$ !)

Due to azimuthal symmetry and longitudinal boost invariance, all mixed components involving indices $\phi$ or $\eta$ of the stress tensor

$$
\sigma^{m n} \equiv \nabla^{\langle m} u^{n\rangle}
$$

and the shear pressure tensor $\pi^{m n}$ vanish such that

$$
\begin{aligned}
& \sigma^{\phi \tau}=\sigma^{\phi r}=\sigma^{\phi \eta}=\sigma^{\eta \tau}=\sigma^{\eta r}=0, \\
& \pi^{\phi \tau}=\pi^{\phi r}=\pi^{\phi \eta}=\pi^{\eta \tau}=\pi^{\eta r}=0 .
\end{aligned}
$$

This leaves only two independent components for the shear pressure tensor which is constrained by the conditions of tracelessness

$$
\pi^{\tau \tau}=\pi^{r r}+r^{2} \pi^{\phi \phi}+\tau^{2} \pi^{\eta \eta}
$$

and of orthogonality to $u^{m}=\gamma_{r}\left(1, v_{r}, 0,0\right)$

$$
\pi^{\tau \tau}=v_{r} \pi^{\tau r}, \quad \pi^{r \tau}=v_{r} \pi^{r r} .
$$

Equations (A6) result from the $n=\tau$ and $r$ components of $u_{m} \pi^{m n}=0$; the other two components yield redundant 
equations. Equations (A5) and (A6) can be combined to yield

$$
\pi^{r r}=-\gamma_{r}^{2}\left(r^{2} \pi^{\phi \phi}+\tau^{2} \pi^{\eta \eta}\right) .
$$

Identical relations hold between the corresponding components of the shear tensor $\sigma^{m n}$. Equations (A5) and (A7) can be used to eliminate $\pi^{\tau \tau}, \pi^{r \tau}$, and $\pi^{r r}$ in favor of $\pi^{\phi \phi}$ and $\pi^{\eta \eta}$, which we keep as independent dynamical variables. Equation (A1) shows that these components are not affected by the radial boost, i.e., the $\phi \phi$ and $\eta \eta$ components of the shear pressure tensor are identical in the global frame and in the local fluid rest frame. The same is not true for the other nonzero components of the shear pressure tensor.

This observation leads to a particularly simple structure of the energy-momentum tensor in the global frame, including viscous pressure terms. Using Eqs. (A5)-(A7) in the expressions for $T^{\tau \tau}$ and $T^{\tau r}$ (which are the two independent components being evolved by the hydrodynamic evolution equations), we find

$$
\begin{aligned}
T^{\tau \tau} & =(e+p+\Pi) \gamma_{r}^{2}-(p+\Pi)+\pi^{\tau \tau} \\
& =\left(e+\mathcal{P}_{r}\right) \gamma_{r}^{2}-\mathcal{P}_{r}, \\
T^{\tau r} & =(e+p+\Pi) \gamma_{r}^{2} v_{r}+\pi^{\tau r}=\left(e+\mathcal{P}_{r}\right) \gamma_{r}^{2} v_{r},
\end{aligned}
$$

where

$$
\mathcal{P}_{r}=p+\Pi-r^{2} \pi^{\phi \phi}-\tau^{2} \pi^{\eta \eta} .
$$

Equations (A8) are the same expressions as those for the ideal fluid, except for the replacement of $p$ by the effective radial pressure $\mathcal{P}_{r}$ which has no explicit dependence on the flow velocity $v_{r}$.

In the local rest frame, the energy-momentum tensor is diagonal:

$$
\hat{T}^{m n}=\left(\begin{array}{cccc}
e & 0 & 0 & 0 \\
0 & \mathcal{P}_{r} & 0 & 0 \\
0 & 0 & \frac{p+\Pi+r^{2} \pi^{\phi \phi}}{r^{2}} & 0 \\
0 & 0 & 0 & \frac{p+\Pi+\tau^{2} \pi^{\eta \eta}}{\tau^{2}}
\end{array}\right) .
$$

The nonvanishing components of the shear tensor $\sigma^{m n}=$ $\nabla^{\langle m} u^{n\rangle}$ are found (after some algebra which properly takes into account the Christoffel symbol contributions to the covariant derivatives) to be

$$
\begin{aligned}
\sigma^{\eta \eta} & =\frac{1}{\tau^{2}}\left(\frac{\theta}{3}-\frac{\gamma_{r}}{\tau}\right), \\
\sigma^{\phi \phi} & =\frac{1}{r^{2}}\left(\frac{\theta}{3}-\frac{\gamma_{r} v_{r}}{r}\right), \\
\sigma^{r r} & =-\gamma_{r}^{2}\left(\frac{2 \theta}{3}-\frac{\gamma_{r}}{\tau}-\frac{\gamma_{r} v_{r}}{r}\right) \\
& =\gamma_{r}^{2}\left(\frac{\theta}{3}-\partial_{\tau} \gamma_{r}-\partial_{r}\left(\gamma_{r} v_{r}\right)\right), \\
\sigma^{\tau \tau} & =\left(1-\gamma_{r}^{2}\right)\left(\frac{2 \theta}{3}-\frac{\gamma_{r}}{\tau}-\frac{\gamma_{r} v_{r}}{r}\right), \\
\sigma^{\tau r} & =v_{r} \sigma^{r r} .
\end{aligned}
$$

Note that (A11b) differs from the corresponding result (44) in Ref. [8] by a metric factor $r^{2}$, and that the last expression in Eq. (A11c) has an extra factor $\gamma_{r}^{2}$ when compared to Eq. (43) in Ref. [8].

\section{Systems without azimuthal symmetry}

Without azimuthal symmetry, there is no advantage in using transverse polar coordinates, and it is simpler to use Cartesian coordinates in the transverse plane: $x^{m}=(\tau, x, y, \eta)$. The global frame and the local fluid rest frame are now related by a Lorentz boost with velocity $\boldsymbol{v}_{\perp}=v_{x}(x, y, \tau) \boldsymbol{e}_{x}+$ $v_{y}(x, y, \tau) \boldsymbol{e}_{y}=\left(v_{x}, v_{y}\right)$, described by the Lorentz transformation matrix

$$
\Lambda_{n}^{m}{ }_{n}\left(\boldsymbol{v}_{\perp}\right)=\left(\begin{array}{cccc}
\gamma_{\perp} & \gamma_{\perp} v_{x} & \gamma_{\perp} v_{y} & 0 \\
\gamma_{\perp} v_{x} & 1+\left(\gamma_{\perp}-1\right) \frac{v_{x}^{2}}{v_{\perp}^{2}} & \left(\gamma_{\perp}-1\right) \frac{v_{x} v_{y}}{v_{\perp}^{2}} & 0 \\
\gamma_{\perp} v_{y} & \left(\gamma_{\perp}-1\right) \frac{v_{x} v_{y}}{v_{\perp}^{2}} & 1+\left(\gamma_{\perp}-1\right) \frac{v_{y}^{2}}{v_{\perp}^{2}} & 0 \\
0 & 0 & 0 & 1
\end{array}\right) .
$$

The projector transverse to the flow vector $u^{m}$ takes the form

$$
\Delta_{n}^{m}=\gamma_{\perp}^{2}\left(\begin{array}{cccc}
-v_{\perp}^{2} & v_{x} & v_{y} & 0 \\
-v_{x} & 1-v_{y}^{2} & v_{x} v_{y} & 0 \\
-v_{y} & v_{x} v_{y} & 1-v_{x}^{2} & 0 \\
0 & 0 & 0 & 1
\end{array}\right)
$$

In the local rest frame, the energy-momentum tensor reads

$$
\hat{T}^{m n}=\left(\begin{array}{cccc}
e & 0 & 0 & 0 \\
0 & p+\Pi & 0 & 0 \\
0 & 0 & p+\Pi & 0 \\
0 & 0 & 0 & \frac{p+\Pi}{\tau^{2}}
\end{array}\right)+\left(\begin{array}{cccc}
0 & 0 & 0 & 0 \\
0 & \hat{\pi}^{x x} & \hat{\pi}^{x y} & 0 \\
0 & \hat{\pi}^{x y} & \hat{\pi}^{y y} & 0 \\
0 & 0 & 0 & \hat{\pi}^{\eta \eta}
\end{array}\right) .
$$

The shear pressure tensor $\hat{\pi}^{m n}$ is no longer diagonal. It may be useful to have expressions for the shear pressure tensor components $\hat{\pi}^{m n}$ in the local rest frame in terms of the hydrodynamic solution for the energy-momentum tensor $T^{m n}$ in the global frame. To this end, we follow Ref. [8] and introduce the mutually orthogonal rest frame four vectors

$$
\begin{aligned}
\hat{u}^{m}=(1,0,0,0), & \hat{i}^{m}=(0,1,0,0), \\
\hat{j}^{m}=(0,0,1,0), & \hat{h}^{m}=(0,0,0,1 / \tau),
\end{aligned}
$$

with $\hat{u} \cdot \hat{u}=1, \hat{i} \cdot \hat{i}=\hat{j} \cdot \hat{j}=\hat{h} \cdot \hat{h}=-1$, such that

$$
\begin{aligned}
\hat{\pi}^{x y} & =\hat{i}_{m} \hat{T}^{m n} \hat{j}_{n} \equiv(i \cdot T \cdot j), \\
\hat{p}+\Pi & =\frac{1}{3}[(i \cdot T \cdot i)+(j \cdot T \cdot j)+(h \cdot T \cdot h)], \\
\hat{\pi}^{x x} & =(i \cdot T \cdot j)-p-\Pi \\
& =\frac{2}{3}(i \cdot T \cdot i)-\frac{1}{3}((j \cdot T \cdot j)+(h \cdot T \cdot h)), \\
\hat{\pi}^{y y} & =\frac{2}{3}(j \cdot T \cdot j)-\frac{1}{3}((i \cdot T \cdot i)+(h \cdot T \cdot h)), \\
\tau^{2} \hat{\pi}^{\eta \eta} & =\frac{2}{3}(h \cdot T \cdot h)-\frac{1}{3}((i \cdot T \cdot i)+(j \cdot T \cdot j)) .
\end{aligned}
$$

The right-hand sides are Lorentz invariant expressions and thus can be evaluated in any reference frame. The vectors $u^{m}, i^{m}, j^{m}, h^{m}$ in the global frame are obtained by applying 
the Lorentz boost (A12) to the rest frame vectors (A15)

$$
\begin{aligned}
u^{m} & =\gamma_{\perp}\left(1, v_{x}, v_{y}, 0\right), \\
i^{m} & =\left(\gamma_{\perp} v_{x}, 1+\left(\gamma_{\perp}-1\right) \frac{v_{x}^{2}}{v_{\perp}^{2}},\left(\gamma_{\perp}-1\right) \frac{v_{x} v_{y}}{v_{\perp}^{2}}, 0\right), \\
j^{m} & =\left(\gamma_{\perp} v_{y},\left(\gamma_{\perp}-1\right) \frac{v_{x} v_{y}}{v_{\perp}^{2}}, 1+\left(\gamma_{\perp}-1\right) \frac{v_{y}^{2}}{v_{\perp}^{2}}, 0\right), \\
h^{m} & =\left(0,0,0, \frac{1}{\tau}\right) .
\end{aligned}
$$

From Eq. (A17d) it follows immediately that the $\eta \eta$ component of $T^{m n}$ is identical in the global and fluid rest frames, i.e., that $\pi^{\eta \eta}=\hat{\pi}^{\eta \eta}$. Longitudinal boost invariance implies that

$$
\sigma^{\eta \tau}=\sigma^{\eta x}=\sigma^{\eta y}=0, \quad \pi^{\eta \tau}=\pi^{\eta x}=\pi^{\eta y}=0 .
$$

The constraints from tracelessness,

$$
\pi^{\tau \tau}=\pi^{x x}+\pi^{y y}+\tau^{2} \pi^{\eta \eta}
$$

and orthogonality to $u^{m}$,

$$
\begin{aligned}
& \pi^{x \tau}=v_{x} \pi^{x x}+v_{y} \pi^{x y}, \\
& \pi^{y \tau}=v_{x} \pi^{x y}+v_{y} \pi^{y y}, \\
& \pi^{\tau \tau}=v_{x} \pi^{\tau x}+v_{y} \pi^{\tau y},
\end{aligned}
$$

then leave us with three independent components of $\pi^{m n}$. Which should we select? From the above it is obvious that $\pi^{\eta \eta}$ should be one of them. For the other two, there are two different possibilities, following from two different chains of reasoning, as explained in Secs. VB 1 and VB 2.

Before giving the explicit expressions for the shear tensor needed in each case, let us first generalize the relations (A8), which are needed to bring the hydrodynamic equations into standard form:

$$
\begin{aligned}
T^{\tau \tau} & =(e+p+\Pi) \gamma_{\perp}^{2}-(p+\Pi)+\pi^{\tau \tau}, \\
T^{\tau x} & =(e+p+\Pi) \gamma_{\perp}^{2} v_{x}+\pi^{\tau x} \\
& =\left(T^{\tau \tau}+p+\Pi-\pi^{\tau \tau}\right) v_{x}+\pi^{\tau x}, \\
T^{\tau y} & =(e+p+\Pi) \gamma_{\perp}^{2} v_{y}+\pi^{\tau y} \\
& =\left(T^{\tau \tau}+p+\Pi-\pi^{\tau \tau}\right) v_{y}+\pi^{\tau y}, \\
T^{x x} & =(e+p+\Pi) \gamma_{\perp}^{2} v_{x}^{2}+p+\Pi+\pi^{x x} \\
& =\left(T^{\tau x}-\pi^{\tau x}\right) v_{x}+p+\Pi+\pi^{x x}, \\
T^{x y} & =(e+p+\Pi) \gamma_{\perp}^{2} v_{x} v_{y}+\pi^{x y}=\left(T^{\tau x}-\pi^{\tau x}\right) v_{y}+\pi^{x y} \\
& =\left(T^{\tau y}-\pi^{\tau y}\right) v_{x}+\pi^{x y}, \\
T^{y y} & =(e+p+\Pi) \gamma_{\perp}^{2} v_{y}^{2}+p+\Pi+\pi^{y y} \\
& =\left(T^{\tau y}-\pi^{\tau y}\right) v_{y}+p+\Pi+\pi^{y y} .
\end{aligned}
$$

If we choose $\pi^{\tau \tau}, \pi^{\eta \eta}$, and $\Delta=\pi^{x x}-\pi^{y y}$ as independent dynamical variables, we can solve the constraints (A19),
(A20) to give the following expressions for the dependent components of $\pi^{m n}$ :

$$
\begin{aligned}
2 v_{x} \pi^{\tau x} & =\pi^{\tau \tau}\left(1+\frac{v_{x}^{2}-v_{y}^{2}}{2}\right)-\tau^{2} \pi^{\eta \eta} \frac{v_{x}^{2}-v_{y}^{2}}{2}+\frac{v_{\perp}^{2}}{2} \Delta, \\
2 v_{y} \pi^{\tau y} & =\pi^{\tau \tau}\left(1-\frac{v_{x}^{2}-v_{y}^{2}}{2}\right)+\tau^{2} \pi^{\eta \eta} \frac{v_{x}^{2}-v_{y}^{2}}{2}-\frac{v_{\perp}^{2}}{2} \Delta, \\
2 v_{x} v_{y} \pi^{x y} & =\pi^{\tau \tau}\left(1-\frac{v_{\perp}^{2}}{2}\right)+\tau^{2} \pi^{\eta \eta} \frac{v_{\perp}^{2}}{2}-\frac{v_{x}^{2}-v_{y}^{2}}{2} \Delta, \\
\pi^{x x} & =\frac{1}{2}\left(\pi^{\tau \tau}-\tau^{2} \pi^{\eta \eta}+\Delta\right), \\
\pi^{y y} & =\frac{1}{2}\left(\pi^{\tau \tau}-\tau^{2} \pi^{\eta \eta}-\Delta\right) .
\end{aligned}
$$

In this approach, we need the following components of the stress tensor $\sigma^{m n}$ as source terms for the kinetic transport equations for $\pi^{\tau \tau}, \pi^{\eta \eta}$, and $\Delta$ :

$$
\begin{aligned}
\sigma^{\tau \tau}= & \frac{\theta}{3}\left(\gamma_{\perp}^{2}-1\right)+\partial_{\tau} \gamma_{\perp}-\frac{1}{2} D\left(\gamma_{\perp}^{2}\right), \\
\sigma^{\eta \eta}= & \frac{1}{\tau^{2}}\left(\frac{\theta}{3}-\frac{\gamma_{\perp}}{\tau}\right), \\
\sigma^{\Delta}= & \sigma^{x x}-\sigma^{y y}=\frac{\theta}{3}\left(2+\gamma_{\perp}^{2}\left(v_{x}^{2}-v_{y}^{2}\right)\right)+\partial_{y}\left(\gamma_{\perp} v_{y}\right) \\
& -\partial_{x}\left(\gamma_{\perp} v_{x}\right)-\frac{1}{2} D\left(\gamma_{\perp}^{2}\left(v_{x}^{2}-v_{y}^{2}\right)\right) .
\end{aligned}
$$

If instead of $\Delta$ we keep $\pi^{\tau x}, \pi^{\tau y}$ as dynamical variables (as explained in Sec. VB 2), we do not need Eqs. (A22a) and (A22b), but we still must express $\pi^{x y}, \pi^{x x}$, and $\pi^{y y}$ [which appear as sources on the right-hand sides of Eqs. (5.20b) and (5.20c)] in terms of those components for which we solve kinetic transport equations. To this end, we rewrite the constraints (A19) and (A20) as

$$
\begin{aligned}
& \pi^{x x}=\frac{1}{v_{\perp}^{2}}\left(v_{y}^{2}\left(\pi^{\tau \tau}-\tau^{2} \pi^{\eta \eta}\right)+v_{x} \pi^{\tau x}-v_{y} \pi^{\tau y}\right), \\
& \pi^{y y}=\frac{1}{v_{\perp}^{2}}\left(v_{x}^{2}\left(\pi^{\tau \tau}-\tau^{2} \pi^{\eta \eta}\right)+v_{y} \pi^{\tau y}-v_{x} \pi^{\tau x}\right), \\
& \pi^{x y}=-\frac{1}{v_{\perp}^{2}}\left(v_{x} v_{y}\left(\pi^{\tau \tau}-\tau^{2} \pi^{\eta \eta}\right)-v_{x} \pi^{\tau y}-v_{y} \pi^{\tau x}\right) .
\end{aligned}
$$

The shear tensor components required on the right-hand sides of Eqs. (5.29) are

$$
\begin{aligned}
\sigma^{\tau x} & =-\frac{1}{2} \partial_{x} \gamma_{\perp}+\frac{1}{2} \partial_{\tau}\left(\gamma_{\perp} v_{x}\right)-\frac{1}{2} D\left(\gamma_{\perp}^{2} v_{x}\right)+\frac{\theta}{3} \gamma_{\perp}^{2} v_{x}, \\
\sigma^{\tau x} & =-\frac{1}{2} \partial_{y} \gamma_{\perp}+\frac{1}{2} \partial_{\tau}\left(\gamma_{\perp} v_{y}\right)-\frac{1}{2} D\left(\gamma_{\perp}^{2} v_{y}\right)+\frac{\theta}{3} \gamma_{\perp}^{2} v_{y} .
\end{aligned}
$$


[1] P. F. Kolb and U. Heinz, in Quark-Gluon Plasma 3, edited by R. C. Hwa and X.-N. Wang (World Scientific, Singapore, 2004), p. 634.

[2] U. Heinz, J. Phys. G: Nucl. Part. Phys. 31, S717 (2005).

[3] C. Eckart, Phys. Rev. 58, 919 (1940).

[4] L. D. Landau and E. M. Lifshitz, in Fluid Mechanics (Pergamon, Oxford, 1963), Sec. 127.

[5] W. Israel, Ann. Phys. (NY) 100, 310 (1976); W. Israel and J. M. Stewart, ibid. 118, 341 (1979).

[6] A. Muronga, Phys. Rev. Lett. 88, 062302 (2002); 89, 159901(E) (2002); Phys. Rev. C 69, 034903 (2004).

[7] D. A. Teaney, J. Phys. G: Nucl. Part. Phys. 30, S1247 (2004).

[8] A. Muronga and D. H. Rischke, nucl-th/0407114 (v2).

[9] A. K. Chaudhuri and U. Heinz, nucl-th/0504022.

[10] D. Rischke, in Hadrons in Dense Matter and Hadrosynthesis, edited by J. Cleymans, H. B. Geyer, and F. G. Scholz, Springer Lecture Notes in Physics, Vol. 516 (Springer-Verlag, New York, 1999), p. 21.
[11] P. Arnold, J. Lenaghan, and G. D. Moore, J. High Energy Phys. 08 (2003) 002; P. Arnold, J. Lenaghan, G. D. Moore, and L. G. Yaffe, Phys. Rev. Lett. 94, 072302 (2005).

[12] J. Berges, S. Borsányi, and C. Wetterich, Phys. Rev. Lett. 93, 142002 (2004); hep-ph/0505182 (Nucl. Phys. B, in press).

[13] A. Rebhan, P. Romatschke, and M. Strickland, Phys. Rev. Lett. 94, 102303 (2005); J. High Energy Phys. 09 (2005) 041.

[14] P. Danielewicz and M. Gyulassy, Phys. Rev. D 31, 53 (1985).

[15] Note that the vector force vanishes in the limit of vanishing net charge $n$ (i.e., zero chemical potential $\mu$ ), so in systems without a conserved net charge there exists no mechanism for generating a heat flow, and heat conduction is zero [14].

[16] J. P. Boris, A. M. Landsberg, E. S. Oran, and J. H. Gardner, LCPFCT-Flux-corrected transport algorithm for solving generalized continuity equations, Naval Research Laboratory Preprint NRL/MR/6410-93-7192 (1993), available at the URL http://www.lcp.nrl.navy.mil/lcpfct/. 